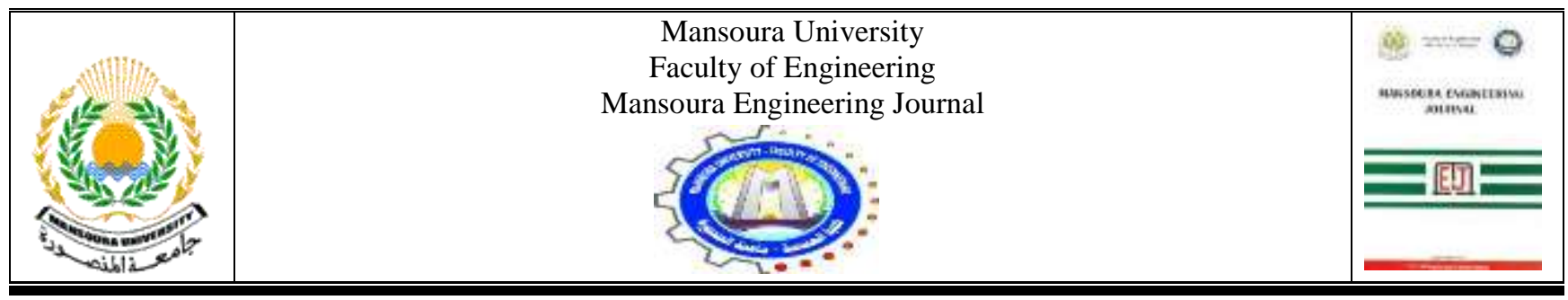

\title{
Flow and Heat Transfer of a Fluid over Stretching Surface with Internal Heat Generation
}

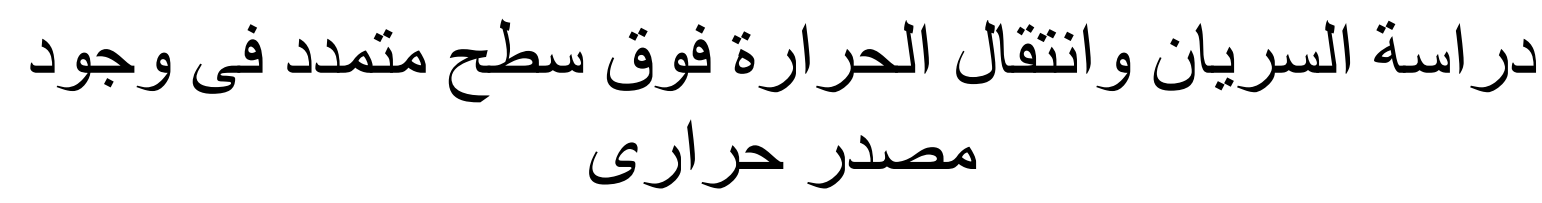

M. Gamal Hassan Wasel, M. Ghassoub Saafan Mousa, Hesham M. Mustafa and Abdelmonaim Fakhry kamel M.

\author{
KEYWORDS: \\ laminar boundary layer, \\ stretching surface, free \\ convection boundary \\ layer
}

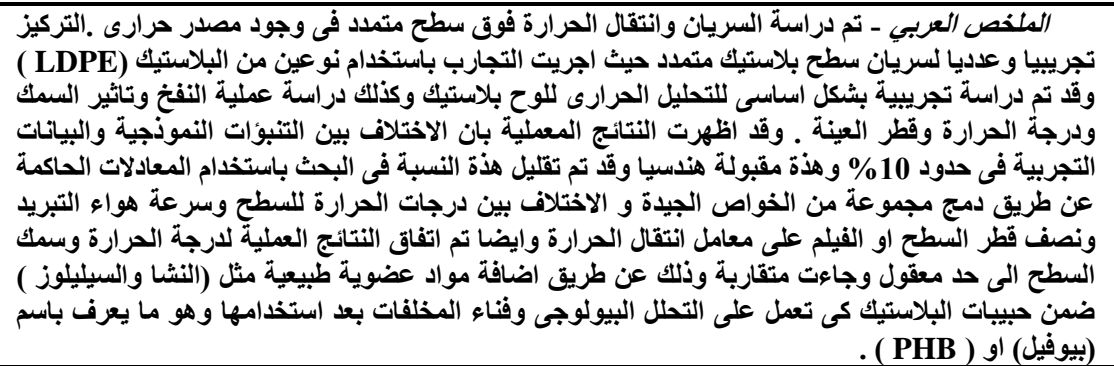

الملخص العريبي - تم دراسة السريان وانتقال الحرارة فوق سطح متمدد فى وجود مصدر حرارى .التركيز

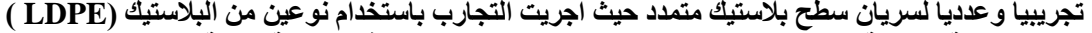

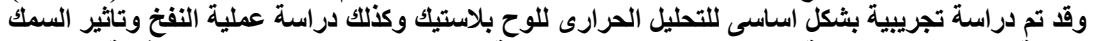

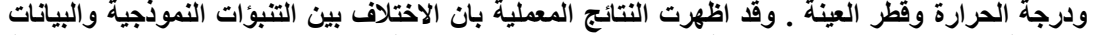
التجربية فى حدود 10\% وهذة مقبولة هندسيا وقد تم تقليل هذة النسبة فى البحث باستخدام المعادلات الحاكمة

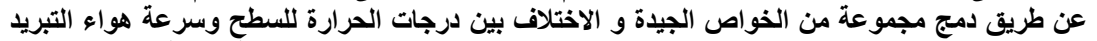

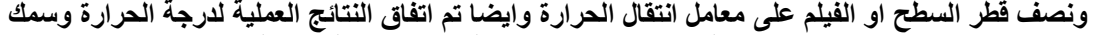
السطح الى حد معقول وجاءت متقاربة وذلك عن طريق اضافة مولى مواد عضوية طبيعية مثل (النشا والسيليلوز )

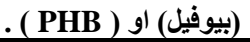

\begin{abstract}
Fluid flow and heat-transfer in a porous medium over a stretching surface have been investigated. The flow is influenced by linearly stretching the surface with internal heat generation or absorption and by the presence of suction, blowing and impermeability of the surface. We have two cases are studied, namely: - (i) Constant surface temperature (CST case), (ii) Prescribed surface temperature (PST case).The effects of Prandtl number, permeability parameter, suction/blowing parameter and heat source/sink parameter on both flow and heat-transfer characteristics are studied and the variations of dimensionless surface temperature with various parameters are graphed and tabulated. In these cases, the final product of desired characteristics depend on the rate of cooling in the process and the process of stretching. Heat transfer in a porous
\end{abstract}

Received: 30 November. 2016 - revised: 16 May, 2017- accepted: 12 June, 2017.

M. Gamal Hassan Wasel,

M. Ghassoub Saafan Mousa (e-mail: mgmousa@mans.edu.eg).

Hesham M. Mustafa , Mechanical Power Engineering, Faculty of Engineering, Mansoura University, Egypt

Abdelmonaim Fakhry kamel $M$. medium over a stretching surface with internal heat generation and suction or injection is studied. The boundary layer equations are transformed to ordinary differential equations containing a Prandtl number, permeability parameter, injection parameter and heat source/sink parameter. Numerical solution for velocity field and the skin friction are obtained. Also, solution for the temperature and the heat transfer characteristics are obtained. Therefore, it is very useful to understand the molecular structure and properties of the polymers before they are selected for processing. Simulation is a useful tool to investigate the process characteristics as well as for scaling up and optimization of the production. And Flow profiles of the blown films. This will help to improve the physical and mechanical properties of the films in a cost effective way, which will in turn be of great benefit to the food and packaging industries. Also the experimental and numerical study of a blown sheet extrusion were carried out using two different low-density polyethylene (LDPEs). In the experiment, the key parameters measured and analyzed were molecular, Time temperature superposition (TTS) technique was utilized to determine the flow activation energy, in the numerical study, blown film simulation was carried out to determine the bubble characteristics and freeze line height (FLH). 


\section{INTRODUCTION}

$\mathrm{T}$ The flow of a viscous fluid over a stretching sheet has important industrial applications, for example, in the extrusion of a polymer sheet from dye or in the drawing of plastic films .during the manufacture of these sheets, the melt issues from a slit and is subsequently stretched to achieve the desired thickness. The final product of desired characteristics strictly depends on the stretching rate. The of cooling in the process and the process of stretching The flow and heat transfer of a viscous and incompressible fluid induced by a continuously moving or stretching surface in a ambient fluid is relevant to the field of chemical engineering processes. Many Mechanical engineering processes like metallurgical process, polymer extrusion process involves cooling of a molten liquid being stretched into a cooling system. In such processes the mechanical properties of the penultimate product would mainly depend on two things, Frist one is the cooling liquid used and second is the rate of stretching., An extreme care has to be given to control the rate at which in place of cooling liquids the extradite is stretched, rapid stretching results in sudden solidification thereby destroying the properties expected for the outcome. The problem addressed here is a fundamental one of practical situations such as polymer extrusion process. These applications include the aerodynamic extrusion of plastic sheets, boundary layers along material handling conveyers, the cooling of an infinite metallic plate in a cooling bath, blood flow problems and textile and paper industries. Impinging on a flat plate, first considered by Sakiadis [1], studied the Boundary layer equation for two dimensional and axisymmetric flows. Sakiadis [2] studied The Boundary layer on a continuous flat surface .Kumaran and Ramanaiah. [3] Studied the flow over a stretching sheet. Ali [4] studied the thermal boundary layer on a power law with suction or injection. Elbashbeshy [5] studied the Heat transfer over an exponential stretching continuous surface with suction. Magyari and, Keller [6] studied the heat and mass transfer in the boundary layer on an exponentially stretching continuous surface. Khan and Sanjayanand. [7]. Sanjayanand. And Khan [8] studied the heat and mass transfer in a viscoelastic boundary layer over an exponential stretching sheet. Andersson [9] studied the MHD Flow of a viscoelastic fluid past a stretching sheet by using shooting method. And also the two dimensional and axisymmetric stagnation point flows with convective heat transfer characteristics over a shrinking sheet. Vajravelu [10] studied the flow and heat transfer in a saturated porous medium. Subhas and Veena [11] studied fluid and heat transfer in a porous medium over a stretching sheet and also the steady two dimensional stagnation point flow of an electrically conducting power law fluid over a stretched surface in the presence of a magnetic field. Eldabe Nabil. and Mohamed Mona. [12] Studied the Non -Newtonian fluid with heat source over an accelerating surface through a porous medium. Cogley et al. [13] studied the Radiation in a non gray gas. Raptis. [14] Studied the mass and heat transfer of hydro magnetic stagnation point flow and heat transfer through a porous space bound end by a permeable surface by using homotopy analysis method (HAM). The influence of radiation on MHD flow and heat transfer problems has received significant importance in industries. Many engineering processes occur at high temperatures and the knowledge of the radiation heat transfer has become important for the design of pertinent equipment. At high operating temperature, the radiation effect can be quite significant. The radiation effects on the flow and heat transfer over a wedge with variable viscosity were discussed and studied by Raptis [15]. Siddheshwar. And Mahabaleshwar [16]. Studied the Effect of Radiation and heat source on MHD flow of a viscoelastic liquid and heat transfer over a stretching sheet. Khan. [17].Recently, Sajid. And Hayat [18] studied the Influence of suction/blowing and thermal radiation on steady boundary layer stagnation point flow and heat transfer towards a shrinking sheet. The magneto hydrodynamic boundary layer flow, heat and mass transfer characteristics on steady two dimensional flow of an electrically conducting fluid over a stretching sheet embedded in a non-Darcy porous medium in the presence of thermal radiation and viscous dissipation was studied by Abel et al [19] studied Flow and heat transfer in a power law fluid over a stretching sheet with variable thermal conductivity and non - uniform heat source. All the researchers cited above are, however, confined to the flow problems of classical Newtonian fluids. The equations of motion for non-Newtonian fluids are highly nonlinear and complicated as compared to those for Newtonian fluids.Chiam. [20] Studied the Heat transfer in a fluid with variable thermal conductivity over a linearly stretching sheet. Predicted experimentally that the fluids having polymeric additives, display a significant reduction of shear stress and polymeric concentration Chiam [21] he studied the heat transfer with variable conductivity in a stagnation - point flow toward a stretching sheet. Chen. Chein [22] he studied the Analytical solution of MHD and types of viscoelastic fluid and thermal radiation. Nag. [23] Studied the heat and mass transfer with stretching sheet. Micro polar fluids have applications in lubricants, liquid crystals, colloidal fluids flow, blood flows, turbulent shear flow and flow in capillaries, heat and mass exchangers etc. The two dimensional non orthogonal stagnation flow of a micro polar fluid on a flat plate was analyzed by Rahman et al [24]. Theoretical analysis of the temperature field in the boundary layer over a stretching sheet were carried out by Dutta and Calcutta [25]. For prescribed sheet temperature distribution or prescribed heat flux by Crane and Dutta [26] reported the analytical solution for cooling of stretching sheet in viscous flow.

\section{MATHEMATICAL MODEL}

Let us suppose a steady laminar and two -dimensional flow of an incompressible fluid through a porous medium of permeability $\mathrm{k}$ over a stretching surface. Two equal and opposite forces are applied along the $\mathrm{x}$ axis so that the wall is stretching keeping the origin fixed. In our analysis we take $\mathrm{x}$ - 
axis along the surface the $y$ - axis being normal to it and $u$ and $\mathrm{v}$ are the tangential velocity and normal velocity, respectively. Thus, for the problem under consideration the equation of the laminar boundary layer is:

$\frac{\partial(\rho \mathrm{u})}{\partial \mathrm{x}}+\frac{\partial(\rho v)}{\partial y}=0$

$\mathrm{u}\left(\frac{\partial \mathrm{u}}{\partial \mathrm{x}}\right)+v\left(\frac{\partial u}{\partial y}\right)=\mu .\left(\frac{\partial^{2} u}{\partial y^{2}}\right)-\{\mu e \cdot(u \backslash k)\}$

u. $\left(\frac{\partial T}{\partial X}\right)+$ v. $\left(\frac{\partial T}{\partial Y}\right)=\alpha_{\mathrm{e}} \cdot\left(\frac{\partial^{2} T}{\partial Y^{2}}\right)+\mathrm{Q} \cdot\left(\frac{\mathrm{T}-\mathrm{T} \infty}{\rho \cdot \mathrm{Cp}}\right)$.

The boundary conditions are:

At $\quad \mathrm{y}=0 \rightarrow \mathrm{f}(0)=1.5 \quad, \quad \mathrm{f}^{\prime}(0)=1$

At $\mathrm{y}=\infty \rightarrow \mathrm{f}^{\prime}(\infty)=0$.

Where $\mathrm{k}$ is the permeability of porous medium. $\rho$ is the density. $\mu$ is the viscosity and $\mu \mathrm{e}$ is the effective viscosity.

One can choose a stream function $\psi(\mathrm{x}, \mathrm{y})$ to satisfy the continuity equations. :

$\mathrm{u}=\left(\frac{\partial \psi}{\partial y}\right)$

$\mathrm{V}=-\left(\frac{\partial \Psi}{\partial \mathrm{x}}\right)$

$\eta=\sqrt{\frac{u \cdot \rho}{\mu}} \cdot y \quad$ And, $\psi(\mathrm{x}, \mathrm{y})=\sqrt{(\mu \cdot u / \rho)} \cdot \mathrm{x} \cdot f(\eta)$.

And substituting in (2) one can get:

$f^{\prime \prime \prime}+f \cdot f^{\prime \prime}-f^{\prime 2}-k_{1} \cdot f^{\prime}=0$.

boundary conditions: $f(0)=\frac{-v w}{\sqrt{u_{\circ}} \cdot v}$

And $, f^{\prime}(0)=1, \quad f^{\prime}(\infty)=0$.

Where $f$ is the non -dimensional stream function. $\eta$ is the similarity variable a prime denotes differentiation with respect to $\eta$. $\mathrm{k}_{1}=(\mu /$ (u. $\rho . \mathrm{k})$ is the permeability parameter and $v$ is the kinematic viscosity. Assuming $f(0)=f \mathrm{w}$ and $f \mathrm{w}$ $>0$ corresponds to suction (i.e. $v<0), f_{\mathrm{w}}<0$ corresponds to blowing (i. e. $v>0$ ) and $f \mathrm{w}=0$ (i. e. $v_{\mathrm{w}}=0$ ) is the case when the surface is impermeable. Assuming that equation (8):

$C=\frac{f \pm \sqrt{f^{2}+4(1+k 1)}}{2}$

Utilizing in section (3) this solution for the function $f$. Stress at a point on the surface is:

$\tau_{\mathrm{o}}=-\mu .\left(\frac{\partial u}{\partial y}\right)_{\mathrm{y}=0}=(\mu$. x.C.u. $) \cdot \sqrt{u / \vartheta}$

The non- dimensional form of shear stress is:

$\tau_{\mathrm{o}}=\left\{\left(\frac{\tau}{\text { u. } \rho . \mathrm{x}}\right)\right\}$

And we obtain from (9):

$\tau=\left\{\left(\frac{\mathrm{C}}{\mathrm{X}}\right) \cdot\left(\frac{\sqrt{\mathrm{v}}}{\mathrm{u}^{\circ}}\right)\right\}$

Being the skin friction coefficient $e=2 \tau$.

From eqs. (4) And (5) we obtain $u(x, y)=u_{o} . x \cdot f^{\prime}(\eta)$.

Where $\mathrm{Cp}$ And $\alpha \mathrm{e}$ are the specific heat of the fluid at constant pressure and the effective thermal diffusivity respectively. Q is the volumetric rate heat generation /absorption.

The boundary condition is satisfy the above eqs. As;

$\mathrm{T}=\mathrm{T}_{\mathrm{w}}$ at $\mathrm{y}=0 \quad, \mathrm{~T}=\mathrm{T}_{\infty} \quad$ as $\mathrm{y} \rightarrow \infty$.

Defining the non - dimensional temperature $\theta(\eta)$ as.

$\theta(\eta)=\left(\frac{T-T \infty}{T w-T \infty}\right)$

And using (4) and (5) in (12), we get.

$\theta^{\prime \prime}+\operatorname{Pr} . f(\eta) . \theta^{\prime}+\lambda . \theta=0$

Where $\lambda$ is: $\quad \lambda=\left(\frac{\sigma . Q}{\rho . C p \cdot U^{\circ}}\right)$

$\lambda$ is Heat source sink parameter. $\operatorname{Pr}=\left(v / \boldsymbol{\alpha}_{\mathrm{e}}\right)=\left(\boldsymbol{\mu} / \boldsymbol{\alpha}_{\mathrm{e} .} \boldsymbol{\rho}\right)$ is the prandtl number primes denote differentiation with respect to $\eta$ and the constants $\mathrm{T}_{\mathrm{w}}$ and $\mathrm{T}_{\infty}$, denotes the temperature at the wall and at a large distance from the wall . Respectively. The boundary conditions for $\theta(\eta)$ follow from (14) and (15) as:

$\theta=1$ at $\eta=0, \theta \rightarrow 0$ as $\eta \rightarrow \infty$.

The rate of heat transfer of the surface is derived from (14) as:-

$\mathrm{q}_{2}=\lambda .\left(\frac{d T}{d Y}\right)$ at $\mathrm{Y}=0 \quad(0)$.

Can be obtained for arbitrarily chosen values of $\left(\frac{d \theta}{d \eta}\right)=\theta^{\prime}(0)$, at $\eta=0$

Assuming that a special value of $\left|\theta^{\prime}(0)\right|$ yields a solution for which $\theta(\eta), \theta$ ' $(\eta)$ Vanishes at a certain $\eta=\eta \infty$. And satisfies the additional condition.

$\left(\frac{d \theta}{d \eta}\right)=0, \theta=0 \quad$ at $\eta=\eta_{\infty}$.

$\theta^{\prime}(0)<0$ implies that the heat flows from the stretching sheet to the ambient fluid (i.e. $\mathrm{T}_{\mathrm{w}}>\mathrm{T}_{\infty}$ ) and in accordance with eq. (14) a negative $\theta$ is not realistic . Consequently for a physical consistent numerical result the corresponding $\theta$ is everywhere finite and non-negative.

Here, the boundary condition is:-

$\mathrm{T}_{\mathrm{w}}=\left(\mathrm{T}_{\infty}+\mathrm{A}\right)$ at $\mathrm{y}=0$.

And $\mathrm{T} \rightarrow \mathrm{T}_{\infty}$ as $\mathrm{y} \rightarrow \infty$.

Where $\mathrm{A}$ is a constant some assumptions concerning the physical validity of the temperature profiles will be necessary .when the wall temperature $\mathrm{Tw}$ exceeds the free stream temperature $\mathrm{T}_{\infty}$ it is obvious that the temperature $\mathrm{T}$ of the fluid inside the thermal boundary layer satisfies $\mathrm{T}_{\infty} \leq \mathrm{T} \leq \mathrm{T}$ w for any $(0 \leq \eta \leq \infty)$ and then we obtain from the first condition (20) that $(A>0)$ and from eq. (14) that $\theta(\eta) \geq 0$. Then, it is obvious that for a physical consistent numerical result the value of $\theta$ is everywhere finite and non-negative. For the condition that $\mathrm{T}_{\mathrm{w}}<\mathrm{T}_{\infty}$, we have $\mathrm{T}_{\infty} \geq \mathrm{T} \geq \mathrm{T}_{\mathrm{w}}, \mathrm{A}<0$, for all $\theta \geq$ $0, \eta \geq 0$. Using eq. (4), (5), (12), (14) and (18), we get:

$\theta^{\prime \prime}+\operatorname{Pr} \cdot\left(f_{w}+B\right) \cdot \theta^{\prime}+(\lambda) \cdot \theta=0$.

$\theta(0)=1, \theta(\infty) \rightarrow 0$.

Eq. (6) can easily be written as the equivalent first order system:

$\mathrm{W} 1=f, \mathrm{~W} 1^{\prime}=\mathrm{W} 2=f^{\prime} . \mathrm{W} 2^{\prime}=\mathrm{W} 3=f^{\prime \prime} . \mathrm{W} 3^{\prime}=f^{\prime \prime \prime}$ 
M: 4 M. GAMAL HASSAN WASEL, M. GHASSOUB SAAFAN MOUSA, HESHAM M. MUSTAFA AND ABDELMONAIM FAKHRY KAMEL M.

$\mathrm{W} 3{ }^{\prime}=-\mathrm{W} 1 . \mathrm{W} 3+\mathrm{W} 2^{2}+\mathrm{k} 1 . \mathrm{W} 2$

And in accordance with boundary condition as;

$\mathrm{W} 1(0)=f(0)=f_{\mathrm{W}}$. W2 $(0)=f^{\prime}(0)=1$.

$\mathrm{W}_{2}\left(\eta_{\infty}\right)=f^{\prime}(\infty)=0$.

Following section (3.1) we guess W3 (0) and solve problem $\{(24),(25)\}$ when $\mathrm{k} 1=1$ and $f \mathrm{w}=1.5$. The results of the numerical solutions for $f(?)$ and its derivatives are shown in fig. (6). Further, we obtain W3 $(0)=(-2.35078)$. When $\mathrm{k} 1=1$, are given in table. From this table, it is obvious that our numerical results are in excellent agreement with those given by eqs. (8) And (9) and. Realize that in this table the numerical values of $\theta$ and $\theta^{\prime}$ are obtained employing the exact functions $f$ and $f^{\prime}$ given by eq. (8). The changes in $\operatorname{Pr}$ and $\lambda$ do not affect the non - dimensional stream function $f$ or its derivatives.

$$
\begin{aligned}
& \text { Friction factor }\left(\mathbf{C}_{\mathbf{f}}\right): \\
& \mathrm{Cf}=\frac{\tau \mathrm{w}}{(0.5) \cdot \rho \cdot \mathrm{U}^{2}} \\
& \text { From } \eta=\mathrm{y} \cdot(\sqrt{u /(v \cdot \chi)} \\
& C_{f}=2 \sqrt{\operatorname{Re}} \cdot\left(\frac{\partial u}{\partial \eta}\right) \quad \text { Local. } \\
& C_{f}=4 \cdot \sqrt{\operatorname{Re}} \cdot\left(\frac{\partial u}{\partial \eta}\right) \quad \text { average. }
\end{aligned}
$$

\section{Nu (Nusselt Number):}

$N u=\sqrt{R e} 2 \cdot\left(\frac{\partial u}{\partial \eta}\right)=0.332 \cdot P^{.0 / 3} \cdot R^{o .3}$

\section{EXPERIMENTAL SET UP}

This section describes the experimental and data acquisition techniques from various equipment's used in this study. The working principle of the instruments will be discussed along with their limitations of measurements. The general steps followed during the experimental stage of this study are: (1) Determination of the thermal and properties of the LDPEs. (2) Determination of the blown film properties, Figure $1 \mathrm{~b}$ ) show the velocity of the film by equation: $\mathrm{V}=\mathrm{S} / \mathrm{t}$. Where $(\mathrm{t})$ is time, $(\mathrm{s})$ distance

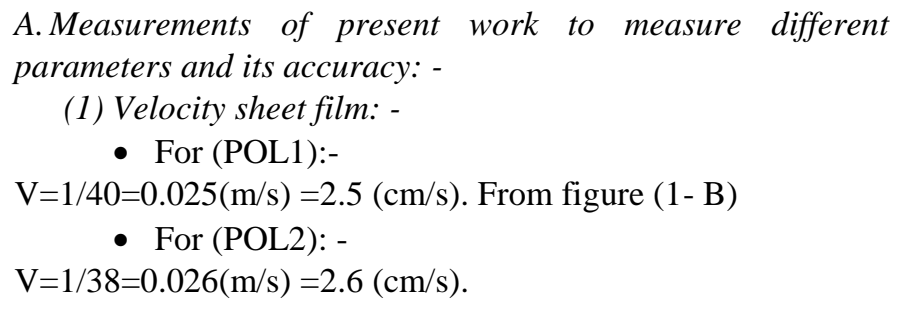

(2) Thickness of the film: -

By Micrometer with Dust/Water Protection Conforming to IP65 Level from figure (1-D): -

- IP65 protection level, enabling use in environments exposed to cutting oil, etc.

- Measurement data output function is available with a water-resistant connection cable.

- Oil-resistance materials are used in all plastic components.

- Instrumental error $(75 \mathrm{~mm} / 3$ ?? or less range models $)$

- Auto power ON/OFF function.

(3) Temperature of the film: -

By Non-contact industrial Infrared Thermometer From figure (1-C): -

This infrared thermometer is used for measuring the temperature of the object's surface, which is applicable for various hot, hazardous of hard-to-reach objects without contact safely and quickly. This unit consist of Optics, Temperature sensor signal amplifier, processing circuit and LCD display. The optics collected the infrared energy emitted by object and focus onto the sensor. Then the sensor translates the energy into an electricity signal. This signal will be turned out to be digital shown on the LCD after the signal amplifier and processing circuit.

\section{RESULTS AND DISCUSSION}

The results of the solutions for two values of $\mathrm{k} 1$ and $\lambda$ when the heat generation is present i.e. $(\lambda>0)$ or it is absent i.e. $(\lambda=0)$ with $\sigma=1$ and $\mathrm{fw}=0$ (impermeability of the surface) are shown the numerical results for two values of fw, $\lambda$ and $\mathrm{k} 1$ with $\sigma=1$. And also seen that the fluid temperature is greater when internal generation exists and, as a result, the thermal boundary layer thickness increases. It is also seen that when suction (i.e. $\mathrm{fw}>0$ ) exists $\left[-\theta^{\prime}(0)\right]$ increases and, as a result, the thermal boundary layer thickness decreases while it increases in the injection case (i.e. $\mathrm{fw}<0$ ). From these tables we also observe that for a given position $\eta, \theta(\eta)$ increases as the permeability parameter $\mathrm{k} 1$ increases for all of the cases. Fig. (4) Shows that, for $\lambda>0$, the effects of the injection and the permeability parameter $\mathrm{k} 1$ are to increase the dimensionless temperature $\theta(\eta)$. In other words, the thermal boundary thickness becomes thinner as the permeability parameter k1 decreases. Fig. (5) Shows the changes in the $\theta$ ( $\eta$ ) profiles with changes in and $\mathrm{k} 1$ in the case of suction. Realize the effect's change of $\mathrm{k} 1$ when heat generation exists (i.e.>0). It is shown that the thermal boundary thickness becomes thinner when absorption heat exists i.e. $(\lambda<0)$ and generation heat augments the thermal boundary thickness. Where $\mathrm{s}$ is the surface temperature parameter and $\mathrm{A}$ is a constant. Some assumptions concerning the physical validity of the temperature profiles will be necessary. When the wall temperature $\mathrm{Tw}$ exceeds the free stream temperature $\mathrm{T} \infty \mathrm{It}$ is obvious that the temperature $\mathrm{T}$ of the fluid inside the thermal boundary layer satisfies ( $\infty \infty \mathrm{T} \leq \mathrm{Tw})$ for any $(0 \leq \eta \leq \infty)$ and then we obtain from the first condition (21) that $A>0$ and from Eq. (15) that $\theta(\eta) \geq 0$. Then, it is obvious that for a physically consistent numerical result the value of $\theta$ is 
everywhere finite and non-negative. For the condition that $\mathrm{Tw}<\mathrm{T} \infty$, we have $(\mathrm{T} \infty \geq \mathrm{T} \geq \mathrm{Tw}), \mathrm{A}<0$ and $\theta \geq 0$ for all $\eta \geq$ 0. Fig. (6) Shows that, in the PST case, the behavior of $\theta(\eta)$ and $\left[-\theta^{\prime}(0)\right]$ with changes in fw and $\mathrm{k} 1$ is similar to CST case. The effect of suction is to decrease the dimensionless temperature profiles whereas the effect of injection is to increase these dimensionless temperature profiles. Finally we see that behavior with changes in $\mathrm{s}$ and $\mathrm{k} 1$ and with changes in $\mathrm{s}$ and $\mathrm{k} 1$ from Figs. (7) and (8), respectively. It can be seen from Fig. (8) That there is a temperature overshoot near the surface when $s=-2$. Note the effect's change of $k 1$ in this case. It is observed that temperature at any point near the surface decreases with increase in $\mathrm{k} 1$ but further away from the surface temperature increases with increase in $\mathrm{k} 1$. It is also seen from Fig. (8- 9) that the thermal boundary thickness becomes thinner as the surface temperature parameter $\mathrm{s}$ increases. In the present study, die exit parameters were used as the initial conditions to solve the steady state blown film equations until the FLH parameters are obtained which is more realistic. Identical inputs and numerical schemes have been considered for simulation using both the (Dutta and Calcutta) and (Crane and Dutta) model. Predictions of the bubble diameter and film thickness from these two different models are presented in Figure (11) along with the experimentally measured data. A long neck of the bubble is predicted near the die exit using the (Crane and Dutta) model followed by a rapid expansion near the FLH Figure (11a). A lower value of FLH is also approximated by using the (Crane and Dutta) model. Compared to the (Crane and Dutta) model predictions, the bubble diameter and film thickness predicted by the (Dutta and Calcutta) model agreed very well with the experimental data as shown in Figure (11). Figure (12) shows a sudden drop of film temperature predicted by the (Crane and Dutta) model at a distance of about $20 \mathrm{~cm}$ from the die exit and after this distance bubble size is found to expand. This is unrealistic in the film blowing process. Heat transfer coefficient data is also observed to decrease from die exit to a distance of $20 \mathrm{~cm}$ and then increase at a distance of about 25 $\mathrm{cm}$ Figure (12(b)). This increased value of heat transfer coefficient seems to have a very little or no effect on the film temperature Figure (12a). A real prediction of the film temperature has been obtained from the model which agrees well with the experimental data as shown in Figure (12). (1) The variation between the model predictions and experimental data is about $10 \%$, which is reasonable for engineering needs. (2) The key findings of this modelling and simulation are: Realistic profile of bubble characteristics (diameter, thickness and temperature) Predictions of freezing line height using the die exit data Reasonable agreement of the predicted value with the present and previous study using different materials and die geometry. These types of predictions (FLH and bubble characteristics of the blown film) are useful to the polymeric film industry to develop suitable materials for blown film extrusion with desirable film properties this simulation is simple and robust in predicting the bubble characteristics quickly to meet the engineering need.

The efficiency of blown film extrusion can be improved by minimizing the temperature of the polymer melt. Reduction of the melt temperature causes the melt to require less heating in the extruder. Normal extrusion conditions have a melting temperature at about $190^{\circ} \mathrm{C}$ despite the fact that the temperature of the melt only needs to be about $135^{\circ} \mathrm{C}$. However, it is not always practical to decrease the melting temperature by that much. By decreasing the melt temperature 2 to $20^{\circ} \mathrm{C}$, the motor load can be decreased by about 1 to $10 \%$. Furthermore, reduction of the melt temperature causes less need for cooling, so there is a reduced use of the cooling system. Moreover, removing heat from the bubble is usually the rate-limiting factor in this extrusion process, so by having less heat in the polymer to remove, the rate of the process can be increased, thus yielding higher productivity. A way to maintain the melt temperature at a minimum is to choose an extruder that is matched to the specific processing conditions, such as the material of the melt and pressure.

Comparison between three study (Crane and Dutta), (Dutta and Calcutta) and (present study):-

In the present study, die exit parameters were used as the initial conditions to solve the steady state blown film equations until the FLH parameters are obtained which is more realistic. Identical inputs and numerical schemes have been considered for simulation using both the (Dutta and Calcutta) and (Crane and Dutta) model. Predictions of the bubble diameter and film thickness from these two different models are presented in Figure (13) along with the experimentally measured data (see Appendix). A long neck of the bubble is predicted near the die exit using the (Crane and Dutta) model followed by a rapid expansion near the FLH Figure (13-a). A lower value of FLH is also approximated by using the (Crane and Dutta) model. Compared to the (Crane and Dutta) model predictions, the bubble diameter and film thickness predicted by the (Dutta and Calcutta) model agreed very well with the experimental data as shown in Figure (13).

Figure (14) shows a sudden drop of film temperature predicted by the (Crane and Dutta) model at a distance of about $20 \mathrm{~cm}$ from the die exit and after this distance bubble size is found to expand. This is unrealistic in the film blowing process. Heat transfer coefficient data is also observed to decrease from die exit to a distance of $20 \mathrm{~cm}$ and then increase at a distance of about $25 \mathrm{~cm}$ Figure (14-b). This increased 
M: 6 M. GAMAL HASSAN WASEL, M. GHASSOUB SAAFAN MOUSA, HESHAM M. MUSTAFA AND ABDELMONAIM FAKHRY KAMEL M.

value of heat transfer coefficient seems to have a very little or no effect on the film temperature Figure (14-a). A real prediction of the film temperature has been obtained from the (crane and dutta) model which agrees well with the experimental data as shown in Figure (14).

\section{CONCLUSION}

Flow and heat transfer in a porous medium over a stretching surface with internal heat generation /absorption and suction or blowing is examined by presenting a similarity solution for the dimensionless stream function and that leads the initial system of five simultaneous ordinary differential equation (ODE) of first order to a system of only two ODEs of first order. With this numerical solution we then solve the equation of the energy numerically .Computation is carried out only for the temperature and the flow characteristics (e.g. Horizontal velocity profiles) are governed by that numerical solution .From that solution and from our numerical results, the following conclusions may be drawn The skin -friction parameter $\alpha=\left|f^{\prime \prime}(0) \quad\right|$ is independent of both the prandtl number Pr and the source / skin parameter $\lambda$. The increase of permeability parameter $\mathrm{k} 1$ leads to the increase of skin friction parameter $\alpha$ in all the cases of suction, blowing and impermeability of the surface. The velocity $u$ decrease with increasing permeability parameter $\mathrm{k} 1$. The effect of suction is to decease the velocity $\mathrm{u}$ whereas the effect of injection is to increase this velocity $u$. The permeability influence on dimensionless temperature profiles has been investigated and.in general the temperature increases with increasing permeability parameter k1. For all of the calculations we have observed that the prantdl number influence is to decrease the temperature with its increases. This is consistent with the fact that the thermal boundary layer thickness decreases with increasing prandtl number $\sigma$. We have also observed that $f^{\prime \prime}(\eta)$ $\rightarrow 0$ as $\eta \rightarrow \infty$.

The variation between experimental and predicted data is about $10 \%$. This variation can be minimized by using the current governing equations for blown film simulation and by considering the following suggestions:

(1) A solution of the blown film process considering film crystallinity and all process dynamics such as bubble instability, freeze line height variation etc. should be attempted.

(2) This study incorporates the effect of temperature difference between the film surfaces, velocity of the cooling air and radius of the bubble on the heat transfer coefficient. However, end effect of the cooling air at the lip of the die exit and after the FLH are also important and needs to be considered for further study in this area.

(3) Die swell and gravity effects can also be considered.

\section{REFERENCES}

[1]. Sakiadis.B.C, Boundary layer behavior on continuous solid surface: I Boundary layer equations for two dimensional and axisymmetric flows, AIChE. J. 7(2007)26-28.

[2]. Sakiadis.B.C, Boundary layer behavior on continuous solid surface: II Boundary layer on a continuous flat surface AIChE.J.7 (2009) 221-225.

[3]. Kumaran.V, Ramanaiah.G, A note on the flow over a stretching sheet, Acta Mech. 116 (2009) 229-233.

[4]. Ali.M.E, on thermal boundary layer on a power law stretched surface with suction or injection Int. J. Heat Mass Flow 16 (2009) 280-290.

[5]. Elbashbeshy.E.M.A, Heat transfer over an exponentially stretching continuous surface with suction, Arch. Mech. 53 (6) (2010) 643-651.

[6]. Magyari. E and, Keller .B, Heat and mass transfer in the boundary layers on an exponentially stretching continuous surface, J.Phys. D Appl. Phys.32 (2010) 577-585.

[7]. Khan.S.K, Sanjayanand.E, Viscoelastic boundary layer flow and heat transfer over an exponentially stretching sheet, Int. J. Heat Mass Transfer 48 (2010) 1534-1542.

[8]. Sanjayanand.E, Khan.S.K, heat and mass transfer in a viscoelastic boundary layer flow over an exponentially stretching sheet, Int. J. Therm. Sci. 45 (2010) 819-828.

[9]. Andersson. H. I, Note: MHD flow of a viscoelastic fluid past a stretching surface, Acta. Mech. (2011); 95:227-30.

[10]. Vajravelu.K, Flow and Heat Transfer in a saturated porous medium, ZAMM 74 (12) 605-614 (2011)

[11]. Subhas.A, Veena.P, Viscoelastic Fluid flow and heat transfer in a porous medium over a stretching sheet, Int. J. Nonlinear Mech. 33 (2011) 531-540.

[12]. Eldabe Nabil.T.M, and Mohamed Mona.A.A, Heat and mass transfer in hydromagnetic flow of the non-Newtonian fluid with heat source over an accelerating surface through a porous medium, Chaos, Solutions and Fractals 13 (2011) 907-917.

[13]. Cogley.A.C, Vincenty.W.E, Gilles.S.E, Differential approximation for radiation in a non-gray gas near equilibrium, AIAA J 1968; 6:551-3.

[14]. Raptis.A, Radiation and viscoelastic flow, Int. Comm. Heat Mass Transfer 26, (6) (2012) 889-895.

[15]. Raptis.A, Technical note: Flow of a micro polar fluid past continuously moving plate by the presence of radiation, Int. J. Heat Mass Transfer 41 (2012) 2865-2866.

[16]. Siddheshwar.P.G, Mahabaleshwar.U.S, Effect of radiation and heat source on MHD flow of a viscoelastic liquid and heat transfer over a stretching sheet, Int. J. Non-Linear Mech. 40 (2012) 807-820.

[17]. Khan.S.K, Heat transfer in a viscoelastic fluid flow over a stretching surface with heat source/sink, suction/blowing and radiation, Int. J. Heat and Mass Transfer 49 (2012) 628-639.

[18]. Sajid.M, Hayat.T, Influence of thermal radiation on the boundary layer flow due to an exponentially stretching sheet, Int. Comm. Heat Mass Transfer 35 (2012)347-356. 
[19]. Abel.M.S, Datti.P.S, Mahesha.N, Flow and heat transfer in a power-law fluid over a stretching sheet with variable thermal conductivity and nonuniform heat source, Int. J. Heat and Mass Transfer 52 (2013) 2902-2913.

[20]. Chiam.T.C, Heat transfer in a fluid with variable thermal conductivity over a linearly stretching sheet, Acta. Mech. 129 (2013) 63-72.

[21]. Chiam.T.C, Heat transfer with variable conductivity in a stagnationpoint flow towards a stretching sheet, Int. Common. Heat Mass Transfer 23 (2013) 239 - 248

[22]. Chen.H-Chein, on the analytical solution of MHD flow and heat transfer for two types of viscoelastic fluid over a stretching sheet with energy dissipation, internal heat source and thermal radiation, Int. J. Heat Mass Transfer 53 (2014) 4264-4273.

[23]. Nag. P.K, Heat and Mass transfer, Tata McGraw-Hill, New Delhi, Ed. Second; 2014.

[24]. 24) Rahman. M.M, Aziz. A, and Al-Lawatia. M.A, Heat transfer in micro polar fluid along an inclined permeable plate with variable fluid properties, I. J. Ther. Sci. 49 (2014) 993-1002.

[25]. 25) Dutta and Calcutta, prescribed sheet temperature distribution or prescribed heat flux. sci.55 (2016)35-65.

[26]. 26) Crane and Dutta, reported the analytical solution for cooling of stretching sheet in viscous flow.42 (2016)3221-3242.

\section{NOMENCLATURE}

Symbol Definition Units

A Cross sectional area of test section

CP Specific heat

$\mathrm{a}, \mathrm{b}, \mathrm{d}$

Width and height and length of test section

$\mathrm{m} 2$ $\mathrm{KJ} / \mathrm{kg} . \mathrm{K}$

$\mathrm{m}$ heat transfer coefficient

$\mathrm{W} / \mathrm{m} 2 . \mathrm{K}$

Thermal conductivity

$\mathrm{W} / \mathrm{m} . \mathrm{K}$

Cf Friction factor

Number of nodes in $\mathrm{x}$-direction

Number of nodes in y-direction

Mass flow rate

Pressure

Heat flux

Function

Perimeter

Temperature

Velocity in $\mathrm{x}$-direction

Velocity in y-direction

Velocity in Z _ Direction

$----$

$-----$

$\mathrm{Kg} / \mathrm{s}$

$\mathrm{N} / \mathrm{m}^{2}$

$\mathrm{W} / \mathrm{m} 2$

$\mathrm{U} / \mathrm{u}$

$\mathrm{m}$

K

$\mathrm{m} / \mathrm{s}$

$\mathrm{m} / \mathrm{s}$

$\mathrm{m} / \mathrm{s}$

Number of finite difference divisions in $\mathrm{x}$ and $\mathrm{y}$ directions, respectively

\section{GREEK SYMBOLS:}

Film thickness

Stream function

Transform axis $\mathrm{x}, \mathrm{y}$

Kinematic viscosity

Dynamic viscosity

Thermal diffusivity

Film thickness

Dimensionless temperature

Density

Shear stress

Subscripts: $\mathrm{x}$

Local L Average \& Main flow w Wall

Dimensionless numbers

$\mathrm{Nu} \quad$ Nusselt Number

Pr Prandtl Number

Re Reynolds Number $(\mathrm{uDh} / \mathrm{n})$

(h.D/K)

$\left(v / \alpha_{e}\right)=\left(\mu / \alpha_{e} . \rho\right)$

\section{$\mathrm{mm}$}

$-----$

$\mathrm{m} 2 / \mathrm{s}$

$(\mathrm{K} / \rho . \mathrm{Cp}) \mathrm{m} 2 / \mathrm{s}$

$\mathrm{m}$

$(\mathrm{T}-\mathrm{Tw}) /(\mathrm{T} \infty-\mathrm{Tw})--\cdot$

$\mathrm{Kg} / \mathrm{m}^{3}$

(N. m-2)

$\mathrm{mm}$

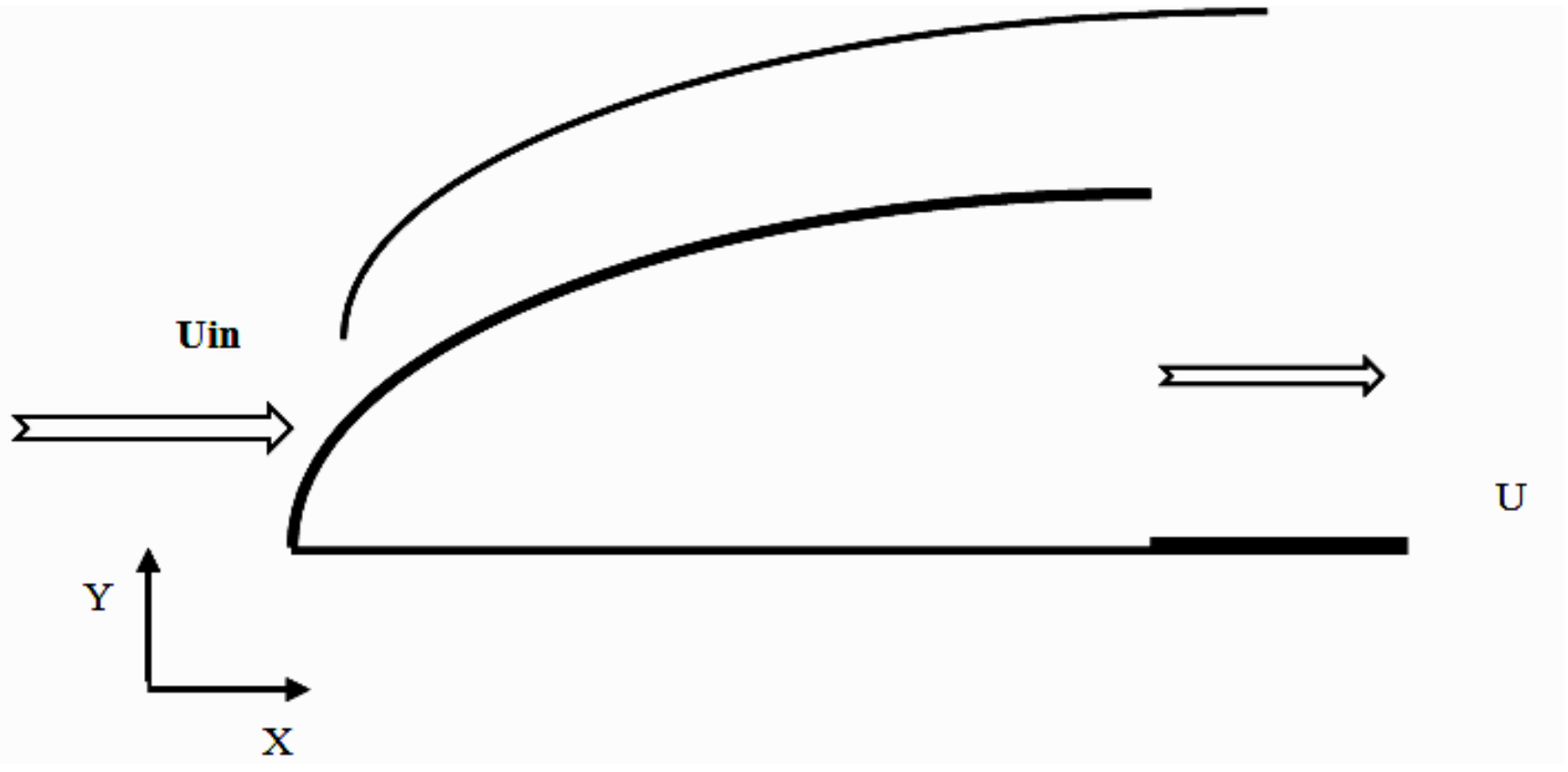

Figure (1-A) scheme of the flow over moving plate 
M: 8 M. GAMAL HASSAN WASEL, M. GHASSOUB SAAFAN MOUSA, HESHAM M. MUSTAFA AND ABDELMONAIM FAKHRY KAMEL M.

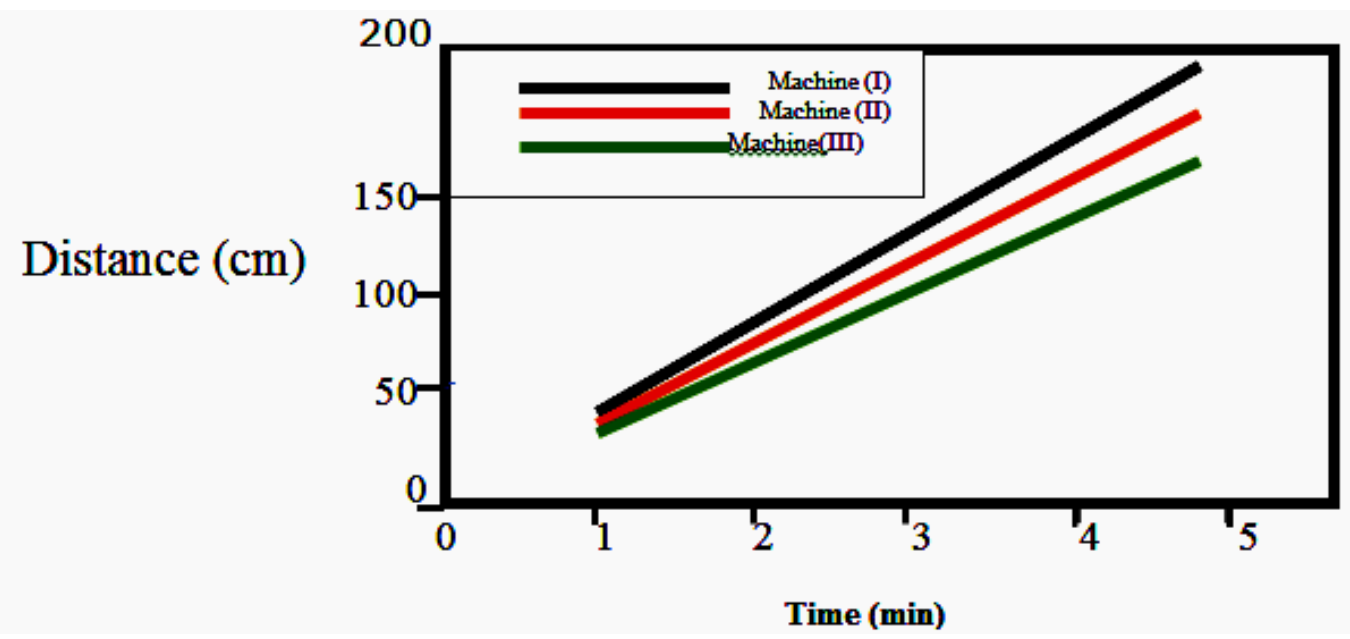

Figure (1-B) show the variation of distance with time for three machine

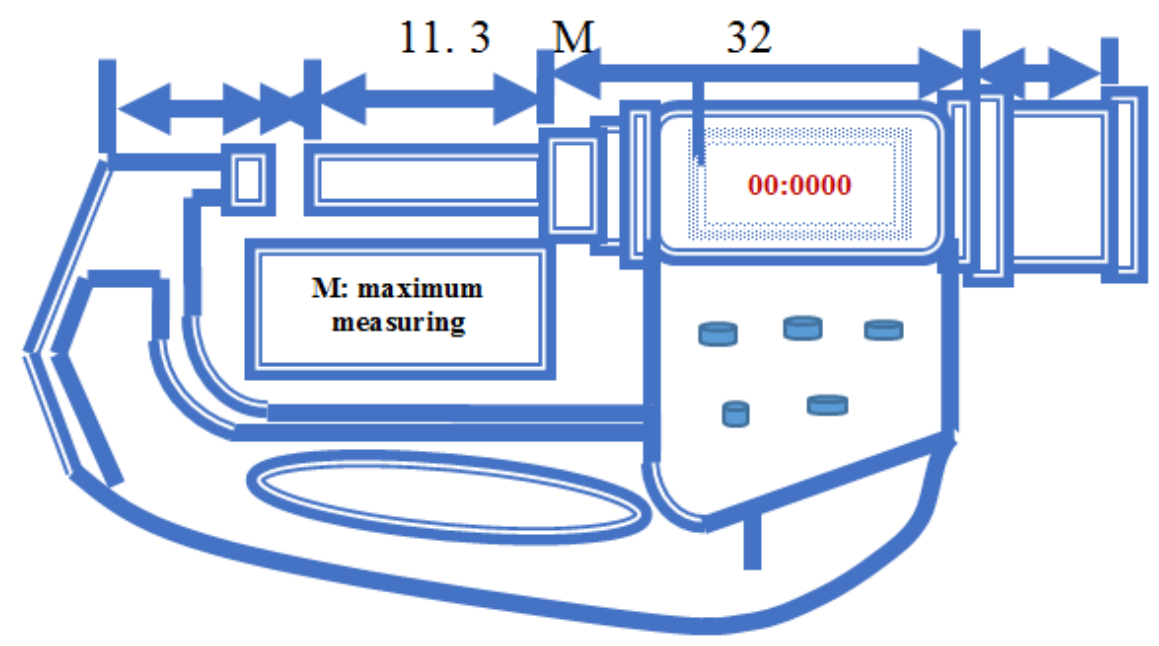

Figure (1-D) mechanism for measuring the film thickness

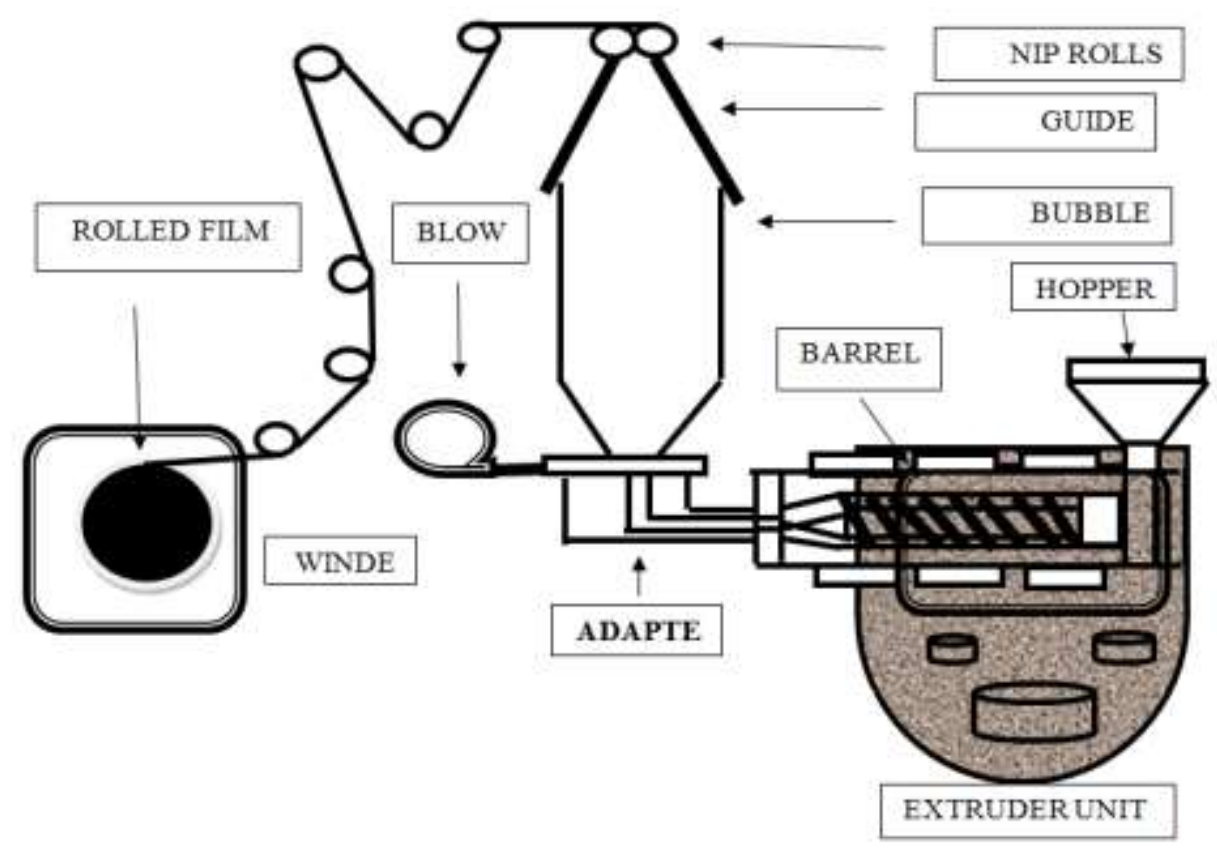

Figure (2) Scheme of the blown film process 


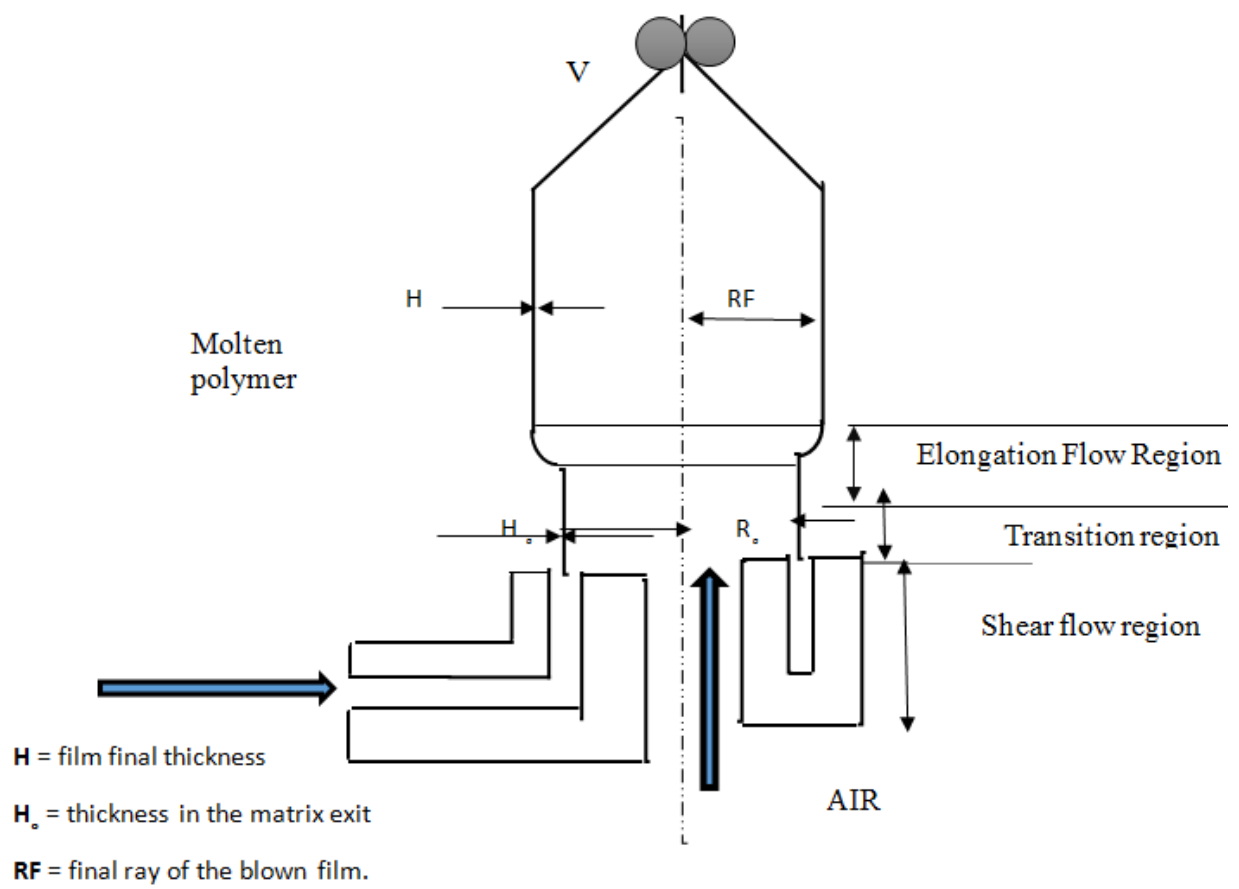

Figure (3) Scheme of the blown film process

$\theta$

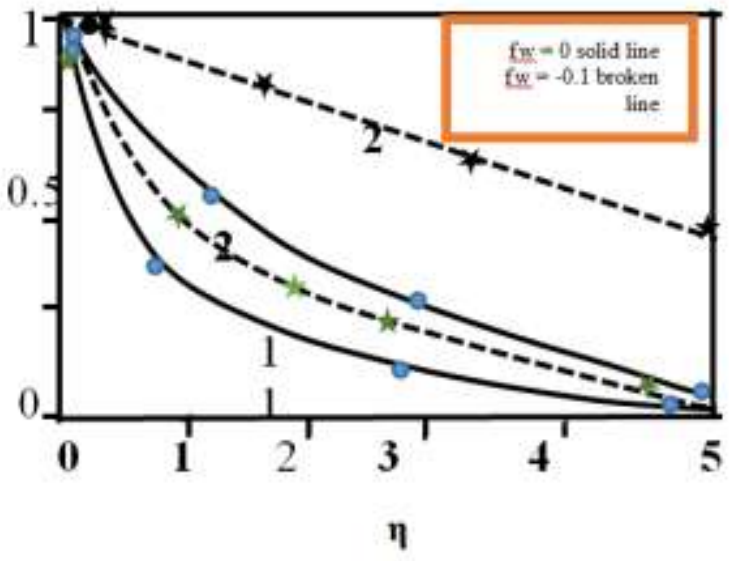

Fig. (4) CST with $\lambda=0.1$

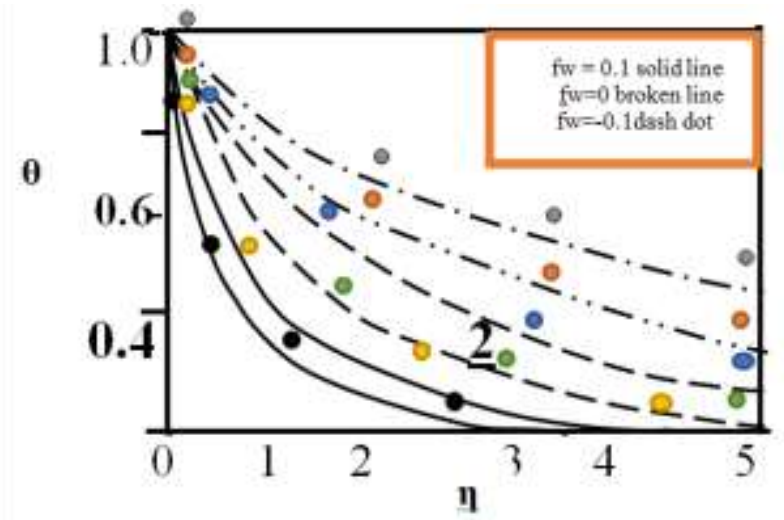

Fig. (6) PST with $\lambda=0.1$ and $\mathrm{s}=2$

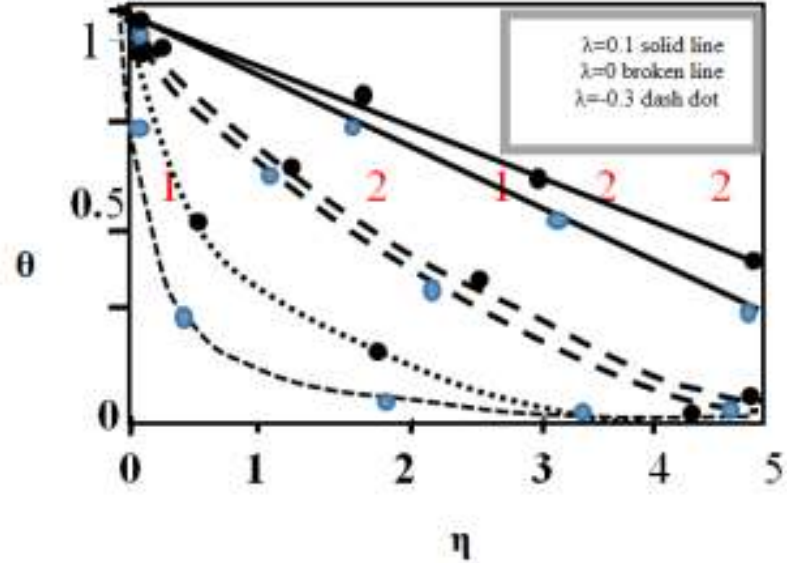

Fig. (5) CST with $\mathrm{fw}=0.1$

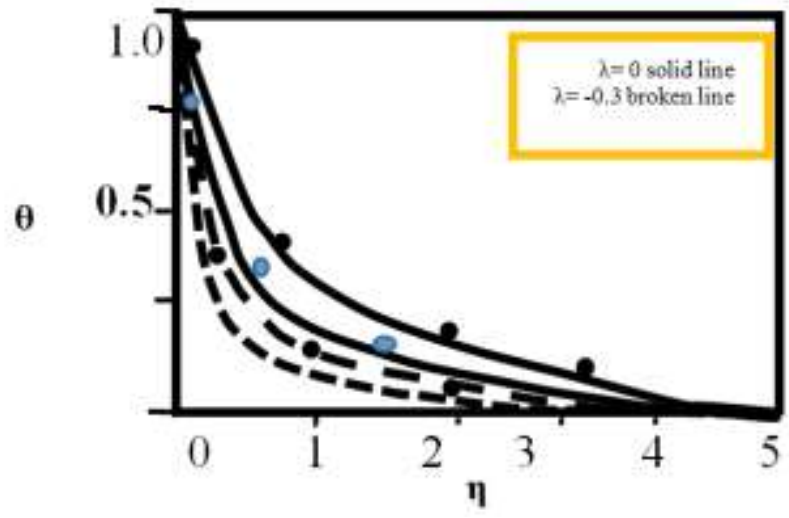

Fig. (7) PST with $\mathrm{fw}=0.1$ and $\mathrm{s}=2$ 


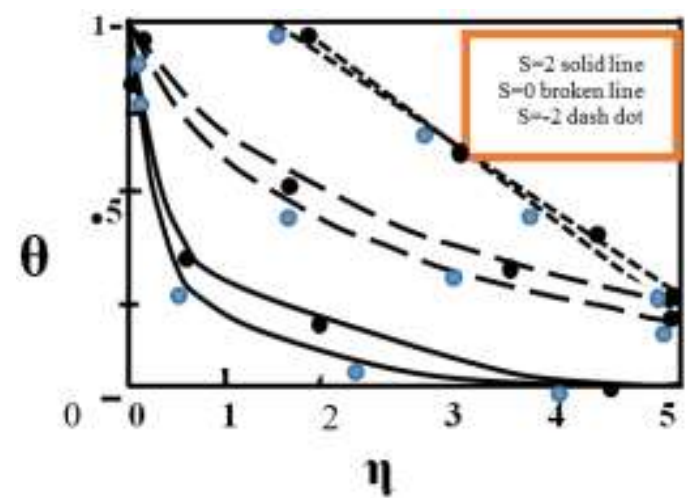

Fig. (8) PST with $w=0.1, \lambda=1$

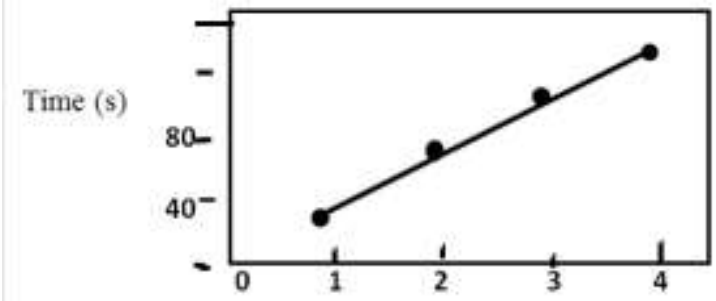

Distance from the die exit

Fig. (10) the distance and the time for bubble film plastic $(\mathrm{m} / \mathrm{s})$

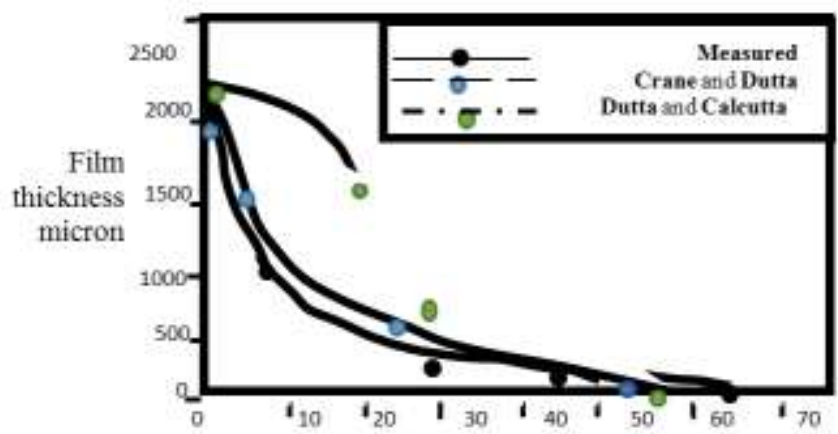

$(11-b)$ Distance from the die exit

Figure (11) : Predictions of blown film characteristics and comparison with the experimental Data, (a) bubble diameter and (b) film thickness

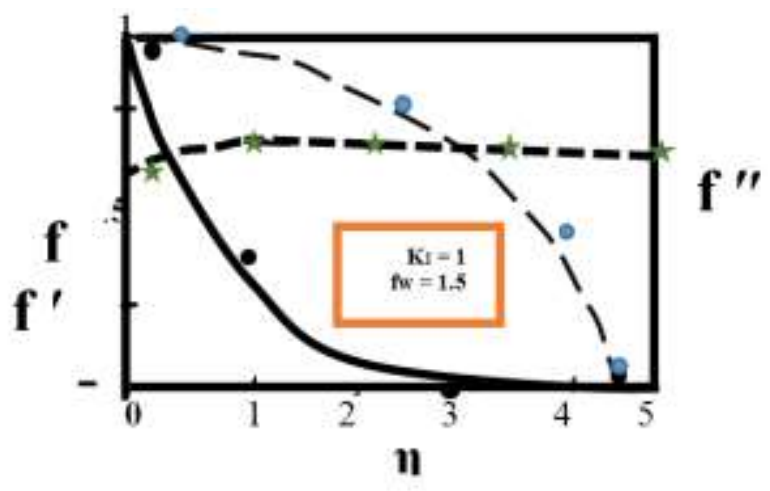

Fig. (9) numerical results momentum poundary laver when fw-1.5

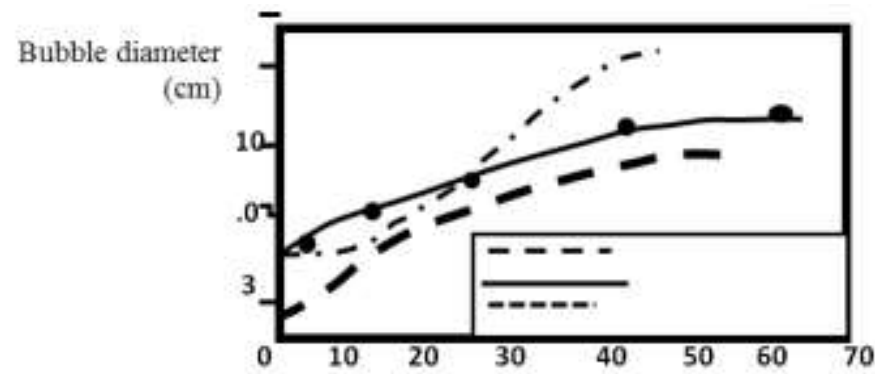

(11-a) Distance from the die exit

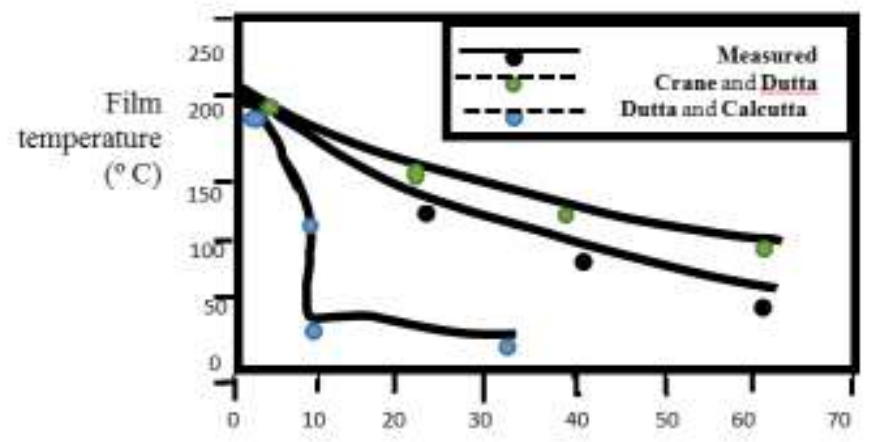

(12 - a) Distance from the die exit 


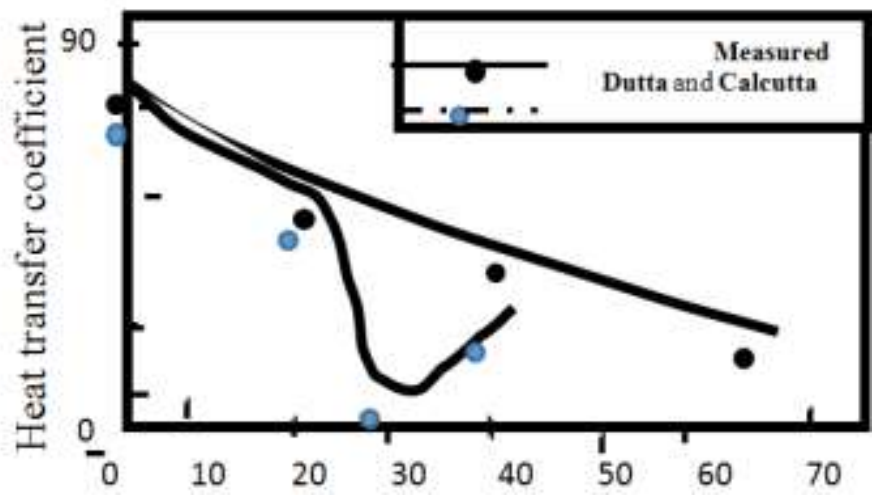

$(12-b)$ Distance from the die exit $(\mathrm{cm})$

Figure (12): Predictions of (a) film temperature and (b) heat transfer coefficient

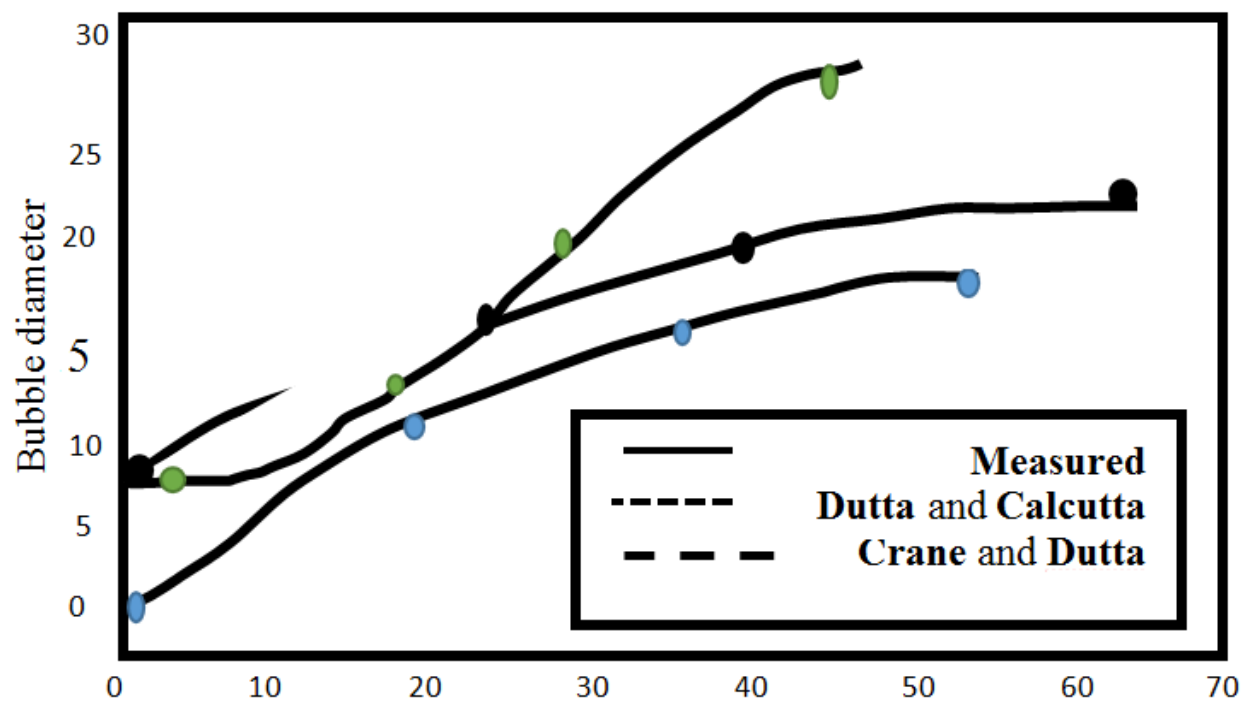

Distance from the die exit

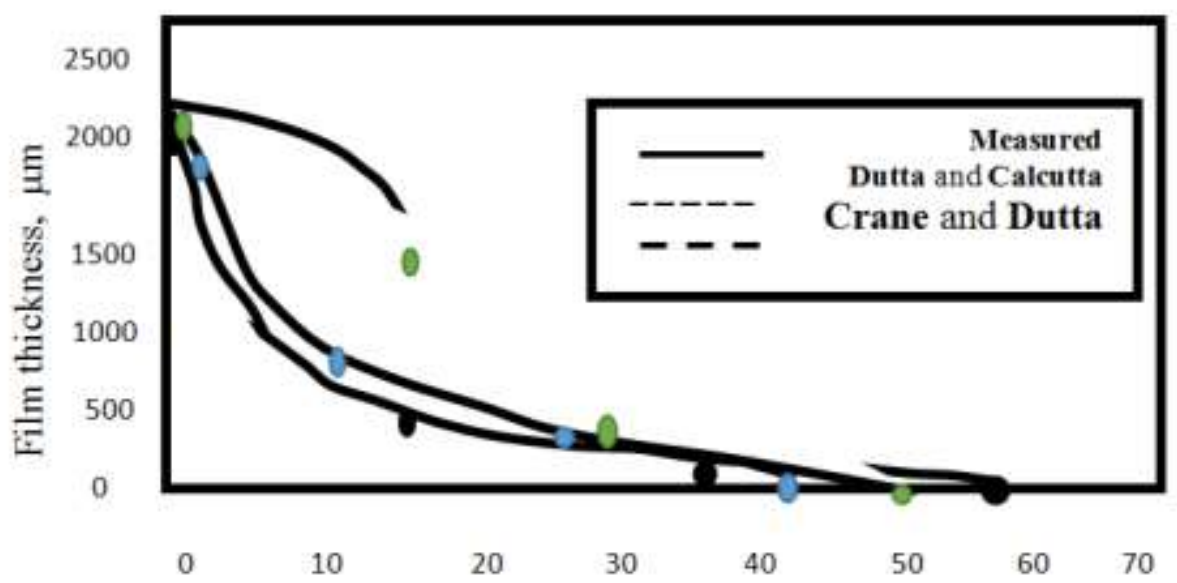

Distance from the die exit $(\mathrm{cm})$

Figure (13) Predictions of blown film characteristics and comparison with the experimental Data, (a) bubble diameter and (b) film thickness 
M: 12 M. GAMAL HASSAN WASEL, M. GHASSOUB SAAFAN MOUSA, HESHAM M. MUSTAFA AND ABDELMONAIM FAKHRY KAMEL M.

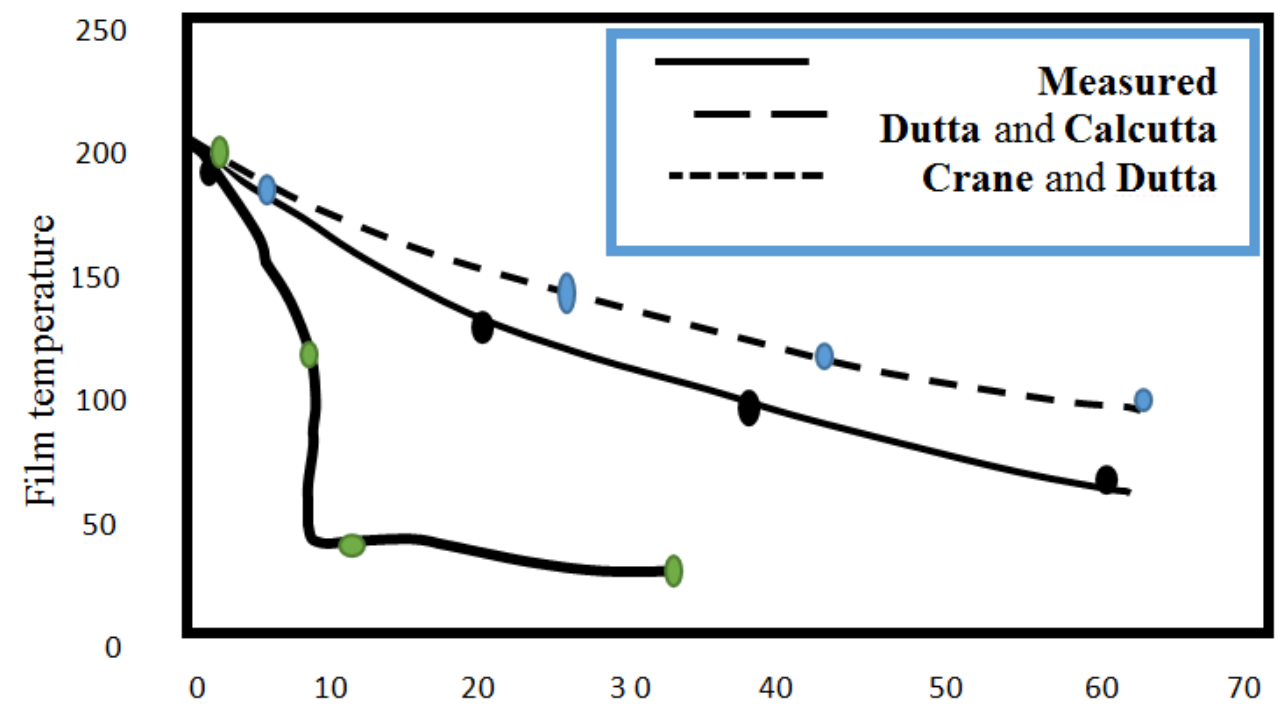

Distance from the die exit $(\mathrm{cm})$

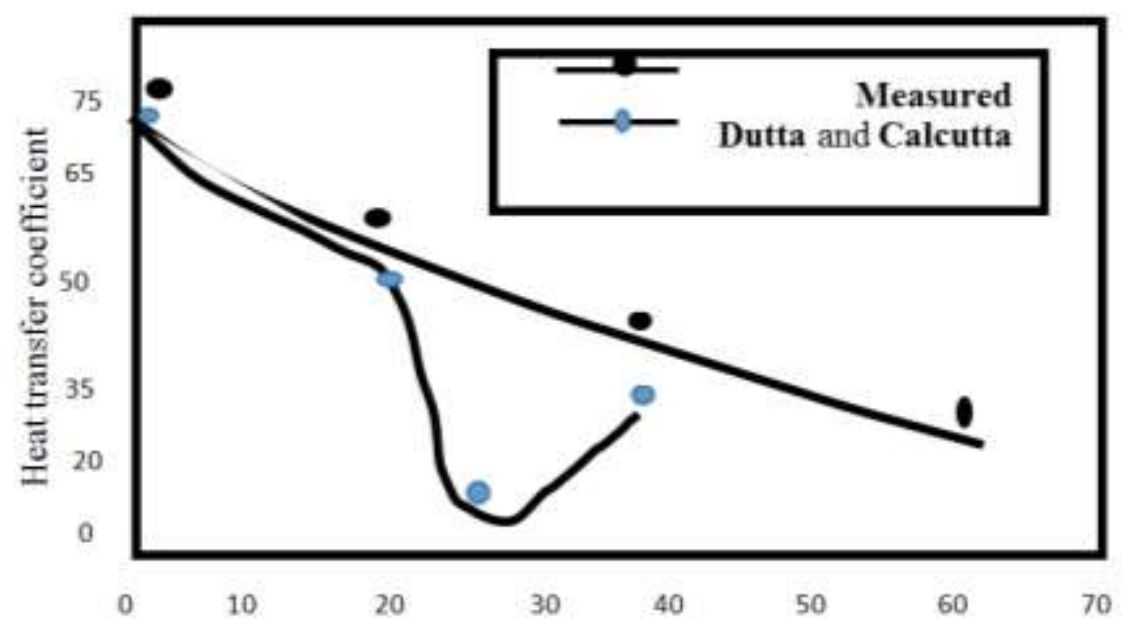

Distance from the die exit $(\mathrm{cm})$

Figure (14) Predictions of film temperature (a) and heat transfer coefficient 